\title{
EMBEDDING IOT SYSTEMS FOR A FLUID POWERED CABLE PULLING WINCH
}

\author{
Kesar M.K. ${ }^{1}$, R. Udayakumar ${ }^{*}$, R. Karthikeyan ${ }^{3}$, Vishweshwar S. ${ }^{4}$ \\ and Nikitha $\mathrm{R}^{5}$ \\ ${ }^{1}$ Department of Mechanical Engineering, Bits Pilani Campus, UAE \\ ${ }^{2}$ Department of Mechanical Engineering, Bits Pilani Campus, UAE \\ ${ }^{3}$ Department of Mechanical Engineering, Bits Pilani Campus, UAE \\ ${ }^{4}$ Department of Mechanical Engineering, Bits Pilani Campus, UAE \\ ${ }^{5}$ Department of Electrical \& Electronics Engineering, Bits Pilani Campus, UAE \\ ${ }^{1}$ kotharikesar@gmail.com, ${ }^{2}$ udaya@ dubai.bits-pilani.ac.in, \\ ${ }^{3}$ rkarthikeyan@dubai.bits-pilani.ac.in, ${ }^{4}$ vishwasamba@gmail.com, \\ ${ }^{5}$ nikitharaj058@gmail.com
}

\begin{abstract}
In this current industrial generation, accustoming to Industry 4.0 or 4th generation automation has been widely recognized. Converting traditional to ergonomically sophisticated machines to function automatically without any intervention of humans have become available in the market. Due to its assurance of efficiency, quality, reliability, and superiority over manual systems, incorporating Industrial Automation has improved productivity within a short period of time. Consequently, upgrading automated machines by integrating a programmable logic controller (PLC) has been a promising solution. Furthermore, this PLC is equipped with a human-machine interface (HMI) which creates a new connectivity method called the Industrial-Internet of Things (I-IoT). This paper focuses on consolidating a completely automated hydraulic machine, the features of IoT and implementing Machine-to-Machine (M2M) Technology. This achieves the main objective of industrial energy saving, occupational safety, labor extensiveness and lowering time consumption by establishing remote control and monitor, remote troubleshoot, feasible technological human interaction and predictive maintenance.
\end{abstract}

Keywords - Automation, Cable Laying, Cellular Mobile Networks, Human Machine Interaction, Hydraulic Winch, I-IoT

\section{INTRODUCTION}

Industrial Automation is a unified sphere of both machines and human-machine interfaces such as PLCs, microcontrollers, remotes etc. The most commonly used specialized controllers are the PLCs; the alternative for traditional relay logic systems. A PLC is a computer to which the user gives an input i.e. processed into a legible output to run a machine. The main parts of a PLC include the chassis, DC/AC power supply, an input, central processing unit (CPU), communication port and an output. These help to control numerous processes like assembling, lifting, transporting, linking, switching and processing data in manufacturing and rugged environment industries. Its robustness makes it easy to control and is generally used in sectors that involve machines that work

Received: January 15, 2019

Reviewed: March 4, 2019

Accepted: March 15, 2019

* Corresponding Author 
under the principle of mechanics, hydraulics, pneumatics, and computerized technology that are utilized individually or combined [1]. Instilling a Human-Machine Interaction (HMI) can only be accomplished by the study of Industrial Internet of Things (I-IoT) with

Machine to Machine (M2M) Technology. It is an innovative emerging type of communication and connectivity i.e. drastically changing the lifestyle of the economy. With the advancements in the modern industrial market and working sectors, interconnecting several devices over a single networking platform with a swipe or touch of a button has become the utmost priority to achieve simplicity and return of investment at a low price. This can help businesses to multiply their productivity, provide a wide exposure in the competitive market, introduce new services and effective modifications, reduce the cost of connectivity, increase energy saving, occupational safety and reduce time consumption and manpower [2]. The implementation of this creative paradigm called I-IoT plus M2M is referred to as Industry 4.0 in which, multiple digitized products that are incorporated with gateways, protocols and sensors can interlink over a network grid called the Internet (Public) or Intranet (Private) and are compatible to remotely transfer, receive, store, monitor and control the inflow and outflow of data using various protocols $[2,3]$.

M2M communication achieves the benefits of wireless connectivity i.e. communication between machines or communication between humans and machines over a distance. In brief, this connectivity allows ubiquitous messaging, data transfer, data feedback, remote control and high-quality connectivity. This solution is given to support the process of cable laying to carefully burying large capacity, high voltage telecommunication, fiber optical and electrical cables in order to achieve a connection between two areas. The hydraulic machine considered in this research is a cable pulling winch; a crucial component of the cable laying procedure. After exhuming a path to lay the cable, this machine is entrenched on one end of the path in order to assist in pulling the cable load from the opposite end. With its basic idea of a pulley system, it assists in unwinding the load from its storage drum. This is simply achieved by attaching the winch rope to the cable load and then providing flow and pressure from the hydraulic system to produce sufficient speed and torque to drive the load bearing modules of the winch. Additional features like sensors, solenoid valves, and linear actuators, also comprise the interior model of the winch which is a closed loop system and provides feedback of the winch parameters. I-IoT is the data exchanged between the sensors in the machine and the PLC whereas; M2M is the link between the human and machine [4]. The organization of this report begins with describing the existing scenario and a solution that can be offered. Materials and methods classify the method of data exchange that discusses about the protocol used and establishing mobile connectivity determines the services needed. Elements in an IoT interface depict the various layers of data flow and PLC as a web server portrays its flexibility. Finally, the results determine the real-time implementation conducted with multiple cases that are observed and solved.

\section{EXISTING PROBLEM AND SOLUTION}

Cable Laying is an effective process that is carried out with the assistance and support of many mechanical and electrical components. The most pertinent module constituting the cable laying procedure apart from its accessories is the Cable Pulling Winch Machine which is a hydraulic machine. The machine considered is completely automated, instigated with solenoid valves, sensors, linear actuators, PLC control panel and a hydraulic closed loop control system. Even with these provisions, a cable laying procedure is cumbersome as it can cover distances of more than $1 \mathrm{~km}$ on harsh site environments. Operators and supervisors risk their safety to oversee the functioning of the machine. Communication between operators and engineers and between operators and the machine becomes difficult as no feedback and diagnosis can be shared between them, 
therefore, no immediate reparations or modifications can be performed. When these winches are transported to other countries, technical assistance can only be provided by proficient staff manufacturing this machine. Travelling proves to be infeasible and impractical to conduct maintenance checks. As per the above literature review, it is observed that the features of IoT have not been incorporated for a specific type of industrial equipment. The results from the approaches such as sensor data analysis, location tagging, cellular/M2M connectivity, feedback control systems, remote control over wireless networks and expedite data transfer have not been holistically considered for a single machine.

Therefore, implementing IoT into this hydraulic machine can bring about modifications in controlling, monitoring, transmitting and receiving data back and forth from the machine. With embedded sensors such as proximity sensors and pressure sensors, feedback of measurements from standard analog or pulse outputs will prove to be useful for operators working on site or in any location. They can understand the working of the machine as well as remotely troubleshoot if any problems are encountered. Selfanalysis and fault diagnosis is conducted to perform predictive maintenance where the staff is alerted about replacements after the runtime of the machine. All this data can be remotely controlled from a distance via a smart phone application and M2M connectivity [5-6]. The PLC used as the control panel in this machine has an internal web server through which the data given to the winch is accessed. PLC's that have inbuilt M2M communication modules or similar features are unavailable in the market as this advancement is relatively new. Thereby, in our research, cases and experiments have been conducted by assembling all these ideas into one hydraulic machine, thus providing the most practical and feasible method to implement human and technology interaction.

\section{METHOD OF DATA EXCHANGE}

This theoretical model of an Open system interconnection (OSI) architecture is considered to be a reference to develop a newer, dynamic, reliable model called the TCP/IP in Figure 1 which consists of 4 layers that are, Application, Transport, Internetwork, Network Access or the Link layer. This makes the implementation of the TCP/IP stack distinct. Its header consists of 20 bytes packet size. A three-way handshake is established before connecting two devices for data transfer. Layer 4 produces a binary code that translates to a protocol data unit (PDU) or the actual data or message that needs to be sent to different devices. Here, this binary code or PDU is encrypted in the form of a file extension to determine the type of data. (eg. .exe, .jpeg, .jpg, .docx, .pdf etc.) PDU's are formed by all layers in the model. This layer majorly determines the transmission, formatting and receiving of data from the other layers or from the user in a network. It follows a sequence of network protocols to do so. Once done, the task is initiated. This 4layer TCP/IP stack assists in standard data exchange, for example, File transfer protocol (FTP) and Hypertext transfer protocol (HTTP).

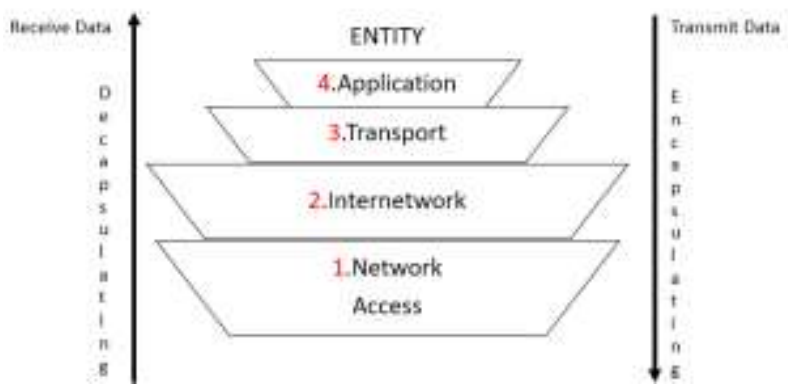

Fig. 1 TCP/IP stack model 
Any device compatible with TCP/IP has a unique physical address engraved into the plating during manufacturing called a hexadecimal Machine Access Code (MAC). This address allows data exchange across devices over a LAN connection. Unfortunately using physical addresses for a wide network with a large data transfer is infeasible due to data traffic. To overcome this problem, logical addressing is adapted which includes the hardware to widen the network and avoid data traffic. Routers disintegrate large networks to small networks called Subnets and it is termed as Routing. These subnets are accessed through logical IP addresses, for example, the host ID is similar to a 12-digit code like 192.168.141.287. The first two terms are called network ID, 3rd term is called the subnet ID and the last represents the host ID. TCP/IP communication directs the routers to choose the practical path for data transfer across the network. Instead of confusing IP addresses, domain names are used as an alternative [6,7]. Domain names are assigned through name servers and are mapped to addresses through Name Resolution. Layer 3 is responsible to decrypt the applications making a request and services that receive them using port addresses. These addresses help the transport layer to assign services to the respective application. This has a source and destination address for every PDU in the transport layer. This PDU is called a Segment. The segment encloses the message that are breakups of data from the entity. At the receiver's end, these segments are recomposed to form a comprehensible message. It allows multiple connections (multiplexing), higher performance, reliability, and security of data transfer. Therefore, the reliable protocol needed to produce the PDU is Transmission control protocol (TCP) and lesser known is User datagram protocol (UDP). The segment is examined and deciphered to send the message to the correct receiver port address. Hence, TCP/IP also performs Port forwarding where ports act as channels to move data, to and from the application layer.

Layer 2 or the internetwork layer identifies an IP address and converts segments to packets of data. An IP also has a source and destination address. This identifies multiple devices interconnected on the network. These packets with the IP address is passed down to Layer 1/ data-link layer plus physical layer/ network access layer in the form of a Frame. The Ethernet protocol containing a source and destination address monitors the connection of modules onto the network and converts all the virtual data to a physical entity, like a signal or an interface card. The physical layer is also considered to be a medium to carry out synchronized transmission between interfaces through coaxial cabling, connectors, fiber optics, Bluetooth, network interface chips, wireless communication like Ethernet etc. It focuses on converting a signal from one type to another to modulate its transfer over a channel. The advantages of TCP/IP make its connectivity reliable as data transmissions are monitored for corruption and loss of data, retransmission possible if data is lost, rearrangement of unclear data, congestion control to delay transmission when network is crowded to avoid packet loss, alerts, when data is received and, transferred, In-band flow control i.e. the rate at which the data is transferred can be varied as well as simultaneously connecting devices in one network. Hence this stream-oriented, high bandwidth process is used for the continuous flow of data during real-time experimentation [8].

\section{ESTABLISHING MOBILE CONNECTIVITY}

The network infrastructure to deliver services between two end nodes to access VoIP and high data transfer is provided by $3 \mathrm{G}$ (third generation, UMTS). This includes a lot of radio equipment and radio access technology (RAT) to ascertain GPRS services. Wide access code division multiplexing (WCDMA) allocates codes to user to differentiate them in the network. The user interface can use the service when a registered SIM is chipped into the equipment.

This authenticates the user to connect to a particular service provider. The users are connected to communication towers or base stations through an Air Interface. These base 
stations conduct transmission and receive radio signals. In a $3 \mathrm{G}$ network, a radio network controller (RNC) supervises base station and user connections or the radio access network (RAN). The base station must take permission from the RNC to process "Hand-over" of data. Followed by the RNC is the pillar of the complete access network called the Code network (CN). The CN consists of Circuit Switched domain (CS) and Packet Switched domain (PS). Here, the user can access the Internet services or PS and voice calling or CS. In detail, the RNC is linked to Serving GPRS support node (SGSN) and Gateway GPRS support node (GGSN). These are in correspondence to GPRS in reference to GSM. SGSN controls the flow of data and GGSN allows the user to get connected to an external data network. An IP network connects both SGSN and GGSN. The bold lines represent. Signaling and data transfer and dotted lines represent signaling interface. Figure 2 depicts the complete architecture of a $3 \mathrm{G}$ network. So, the service that brings in packet data or Internet and voice calling is called as General Packet Radio Services or GPRS. This is a modified GSM architecture as GSM is only used for calling services. This packet has the data information and control information. The control information consists of the source and destination address provided by the user. This helps to transmit the message and retransmit if not received by the receiver. To avoid data mishandling, GPRS also includes error correction bits.

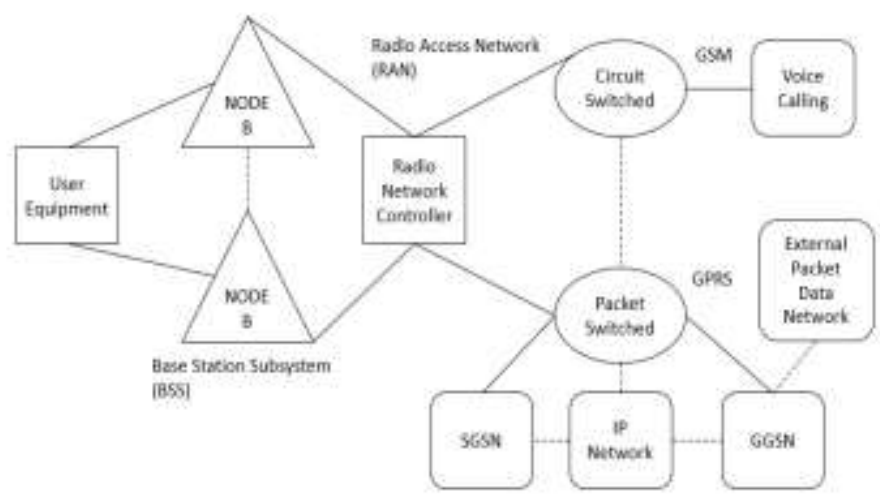

Fig. 2 3G UMTS block architecture

The packet is classified into header, payload and trailer. The source address, data and destination address are stored in the packet respectively. As GSM and GPRS correlate with each other, the length of the packet should be equal to the GSM time slot. The architecture is elaborated in a simplified manner in Figure 3. To begin with, the user equipment receives and transmits data to the Base station subsystem (BSS). The hardware of BSS constitutes of a base transceiver station (BTS) and other modules such as antennas, base station controller (BSC) to exchange and control radio signals. The BSS consists of identification addresses allocated to the equipment and the network its connected to, which is acquired from the Mobile Switching Center (MSC) i.e., interfaced with the Visitor Location Register (VLR). This VLR is utilized when the equipment is in a roaming network i.e., when the connection tower is changed when the user moves from one area to another.

All this data is sent to the SGSN node and the BSS assigns an SGSN node to the user. Here, the data is filtered and compressed as well as it performs user authentication and monitors the function of IP address. This node is also interfaced with Equipment Identity register (EIR); tags to identify users or the mobile equipment identity number (IMEI)on the blocked network list; and Home Location Register (HLR); this stores the change in location when the user switches on roaming services. SGSN requests permission from GGSN to access the packet data network through routing information. 


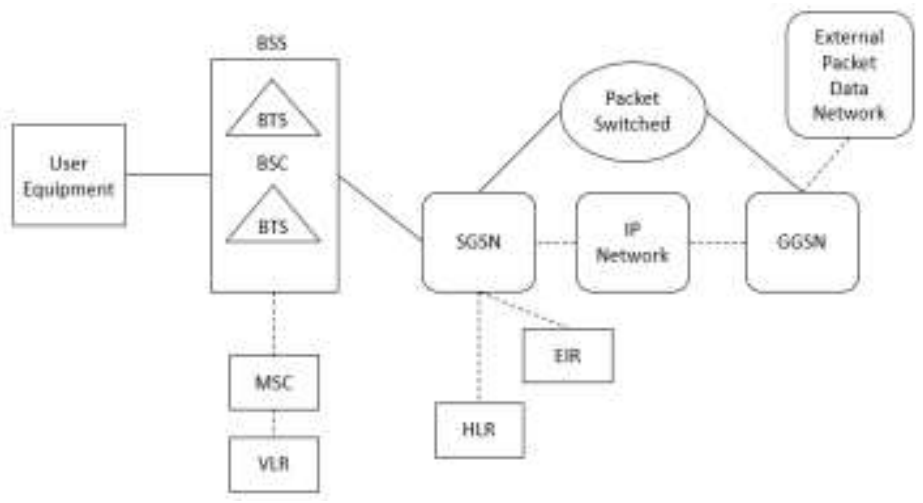

Fig. 3 Architecture of a GPRS system

\section{ELEMENTS OF AN IOT INTERFACE}

As a basic explanation, Figure 4 summarizes the IoT ecosystem and its implementation. The physical layer is the lowest level and the entity is the highest level in the structure. Firstly, the physical layer supports the interface and hardware constituting an IoT device. This layer receives the data collected by sensors present in the machine. It is utilized by the entity to analyze and observe feedback of the parameters from the machine. Therefore, proximity sensors, pressure sensors, load cells networking gear, wiring, and PLC allows data exchange. Next, the network layer assists in bridging the physical layer to the rest of the elements in the system. Here, the network layer is provided by the ISP to perform the function of M2M. It is responsible to transfer and collect the data between machines and devices. Global System for Mobile Communications (GSM used in $2 \mathrm{G}$ networks) and General Packet Radio Services (GPRS) are predominantly used both in mobile telephony communications as well as to instill industrial IoT that requires a wide area network (WAN) and long-range communication.

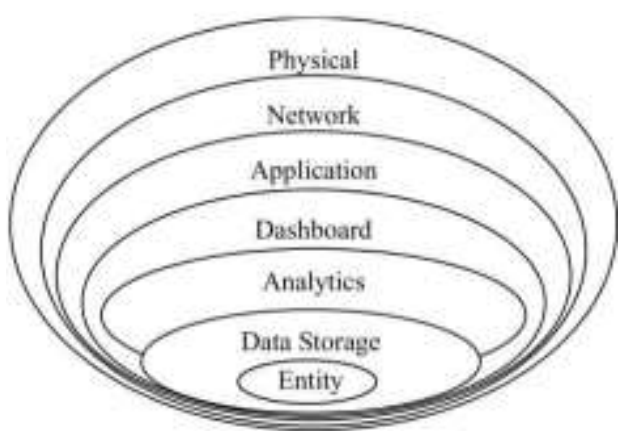

Fig. 4 Elements of the IoT ecosystem

Wireless sensor networks (WSN) can also be embedded in a machine to use Locationbased services (LBS) such as Global Positioning System (GPS) to track the location of the machine using digital maps in case of emergency technical assistance is necessary. Here, RFID isn't preferred as its main disadvantage is radio frequency interference from other frequency channels that can disrupt the communication between operators and supervisors working on site [5,9]. Communication towers are scarce in populated areas as it can ruin the aesthetics of the city. The network layer supervises the connectivity between machines using devices such as cellular routers, cellular modems, antennas, Subscriber identity modules (SIM) cards etc. specifically designed to create a gateway. The application layer encompasses of various procedures like Port Forwarding, Network Address Translation (NAT) using Dynamic Host Configuration Protocol (DHCP) or a 
Static IP address which designates an address to machines to be interconnected. Therefore, it provides the provisions and protocols to accomplish human-digital communication and digital-digital communication accurately. The network and application layer simultaneously function to receive and assign addresses and information to and from the communication carrier service and the manufacturer's machine. The Ethernet protocol is the service used between the cellular router and PLC to provide the Internet packets. In Figure 5 web servers with IP addresses connect the PLC to a smart phone [10]. The dashboard is basically a platform or a screen where the entity or user can observe, simulate, analyze and refer all the information circulated between machines from the preceding three layers and overcome any obstacles encountered. This is practically the HMI i.e., the PLC screen or a mobile application developed.

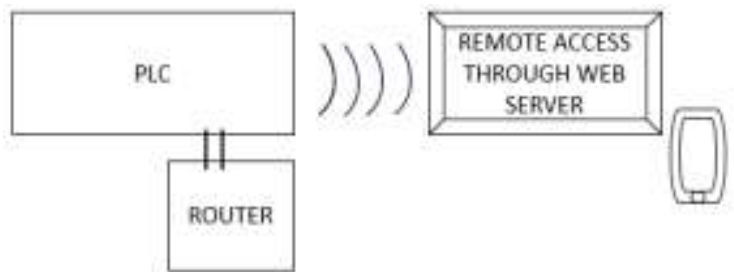

Fig. 5 Brief block diagram of IoT connection

The analytics or the software required to program and configure all the modules outlines the coding section of IoT implementation. Here, Android Studio and Ladder Logic constitute the analytics that programs mobile application in Figure 6 and the PLC in Figure 7 respectively. These figures depict the layouts designed for the display of the PLC placed in the control panel of the hydraulic machine manufactured. A website server is developed specifically for the control of the cable pulling winch machine that easily interprets the functions of the winch using a sequence of buttons and easy mapping for ease of access for new labors. This website server (address) is assigned to the PLC by the ISP and the ladder logic program is dumped into the software of the PLC.

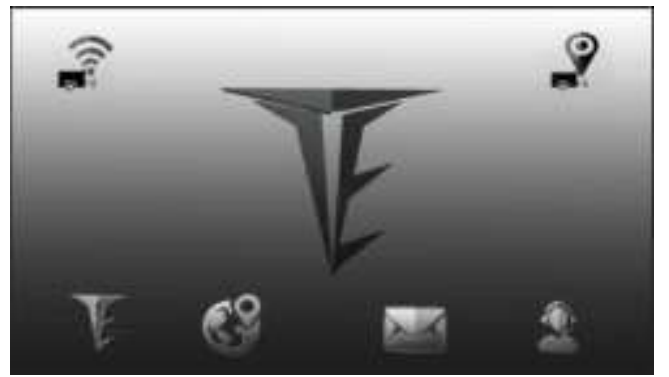

Fig. 6 Layout of mobile application to control winch

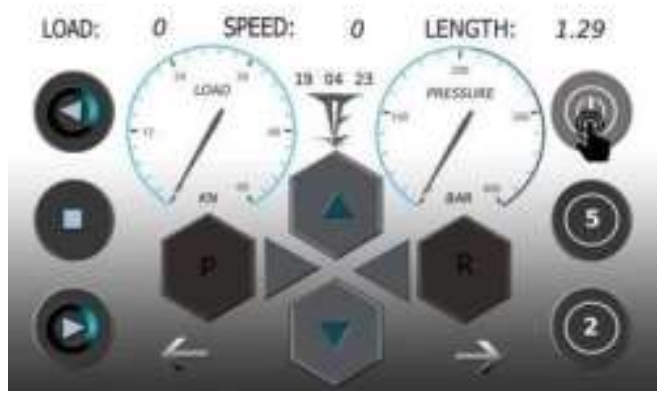

Fig. 7 HMI touch screen designed for the PLC 
Finally, the data inventory or storage is maintained and secured in a cloud storage that is accessible by the organization's eligible staff. Here, data recovery is also possible through thermal printing where the "Print Button" on the PLC screen is clicked to print out a piece of paper that has all the details of the undergoing operation. Also, the company details, the model number of the machine, load capacity, main parameters and the type of operation are also mentioned. The "Record Button" records the history of the winch as well as present processes and this is converted into a document that can be saved in a Universal serial bus (USB) for future references. As mentioned above, the entity is the operator working on site of the company manufacturing the cable pulling winch machine. The entity can also be a business, consumer as well as a government organization.

\section{PLC AS A WEBSERVER}

A PLC is enabled with an inbuilt free port (FP) web server system either by the process of creating a Hypertext machine language (HTML) code editor using HTTP protocol and JAVA language or by purchasing a PLC with a web server enabling system and configuring using the necessary program. This HTTP protocol is a client/server based system where users can extract all data, services, and files from the web interface through a mobile device or desktop connected to a hardwired LAN or WAN or a remote wireless network from any location. This web interface decodes Ethernet LAN TCP/IP and other protocols to interpret data legible to the PLC. The PLC connects to the FTP server via RS232 serial communication to approach the TCP/IP network. Embedding web servers in PLC that requires only a small memory storage and connected onto the network is a simpler alternative for expensive separate cloud base storage systems.

This web server can give real-time continuously flow of data. Any type of web browser is well-suited to access the data as long as there is an internet connection. The IP address of the PLC links to a web server and allows the user to access the program dumped into the PLC as a website when the user inputs the address into the mobile application. Therefore, the operator can retrieve and upload files, adjust graphics according to their requirements, give instructions to function the PLC as well as control and monitor the equipment. Modular PLCs are used when multiple inputs and outputs are needed on the chassis. The outputs and inputs can either be digital or analog such as switches, transducers, regulators, meters, valves etc. A PLC's CPU is configured using a programming code called Ladder Logic. This enables the user to feed a coded program that instructs the $\mathrm{CPU}$ to decide its functions. This staircase resembling program ensures the operation to run in a sequential fashion.

\section{TESTING AND RESULTS}

The cable pulling winch is sophisticated by incorporating IoT technology and is the physical layer of the IoT ecosystem. In detail, the PLC of the winch acts as the physical layer. This IoT is integrated to accomplish three important functions to expose the uniqueness of the winch into the commercial competitive market. These functions include:

a) Remote trouble-shooting by using embedded pressure and proximity sensors that receive feedback from the hydraulic pumps and the corresponding motors that drive Pull In and Pull Out operation of the winch. These feedbacks are values of the required amount of pressure and flow in the hydraulic circuit system.

b) Predictive Maintenance; self-alerting the supervisors and staff on site for assistance when a problem is encountered in the hardware modules of the machine. It notifies the operator to assess the module stock quantity and then cautions the staff to process a module replacement if necessary.

c) Remote control and monitor; using a mobile application and cellular connectivity to 
control and operate the working of a winch from a distance to receive and store data from the winch's PLC for future reference.

In the complete study of the hydraulic circuit, every operating condition and parameter of the system is known to the user. The input parameters given are external load, engine speed and pump displacement. By adjusting these variables, it is observed that the response variables of pressure and speed of the fluid in the system are varied. Therefore, to regulate the hydraulic circuit, the values of input and response variables must match. When these values differ during the run-time of the machine, a problem is encountered. A method to overcome this situation is done by Remote Trouble Shooting. This method is performed when the sensors embedded in the system produce a feedback to alert the operator, the instability in the system. With the experience and engineering qualification, the operator can perform a spontaneous thorough check of the system on site. Proximity and pressure sensors are integrated into the internal structure of the winch machine.

The output and input lines are interlinked to the wiring of the PLC. Digital signals are exchanged between the sensor and the PLC to receive the feedback. It is also possible to toggle between different valves in the system such as sequence valve, check valve, pressure relief valve and directional control valve as well as the type of load (2.5ton, 10ton) and type of mode (automatic, manual). This is mostly caused during Pull In and Pull Out operation. A primary check is completed during Pull In operation to evaluate the whole system of the winch to check the synchronized rotation and RPM of the three motors to drive the capstans and storage drum. When this check fails, the winch remotely troubleshoots. These evaluations are thoroughly performed in [11]. Reference [11], consists of flow charts and algorithms which are used to explain the pull in and pull out operations as well as the toggling between types of loads. The authors also simplify the mechanism on how the PLC acts as the core of the data transfer in the fluid powered machine. Figure 8 depicts the cause of troubleshooting during pull-in operation. The "dash" lines represent sufficient inputs and outputs given, the "double dot dash" line represents insufficiency of the specific parameter and "single dot dash" lines represent cause and effect.

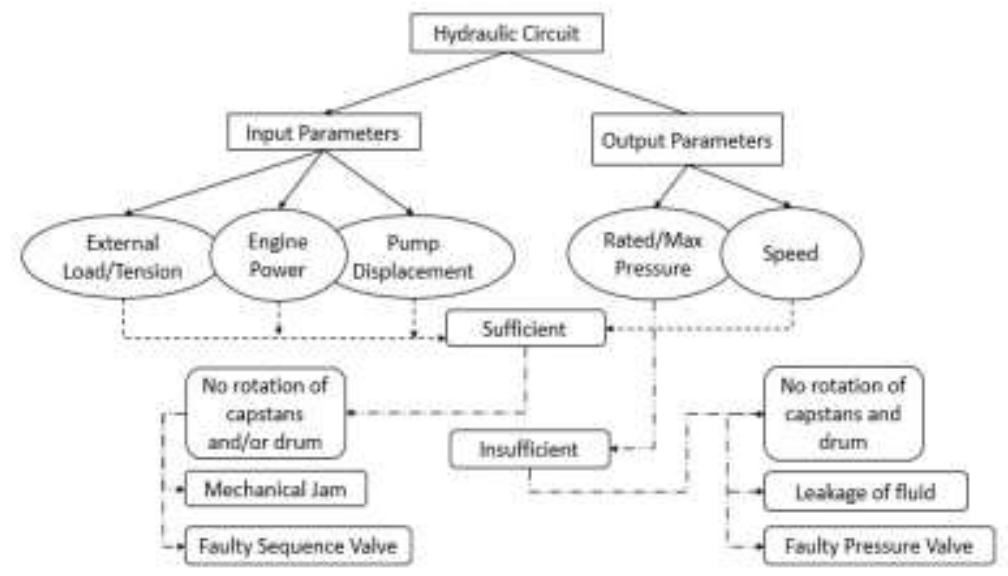

Fig. 8 Causes of troubleshooting during pull in operation

Case 1: When sufficient tension, engine power, pump displacement, speed and pressure is given to the system but the capstans and drum don't rotate then the reasons can be either due to a mechanical jam or a faulty sequence valve. This feedback is provided by the proximity sensors embedded near the motors.

Case 2: When sufficient tension, engine power, pump displacement and speed is provided but insufficient pressure doesn't rotate the capstans and drum, the reason can either be due to a faulty pressure relief valve or due to a leakage of fluid in the system. 
Case 3: The directional control valve can also be monitored while toggling between series and parallel modes i.e., shifting between the load capacities. In the parallel mode, if the inlet pressure of both capstans and storage drum motors aren't equal then the control valve is justified as faulty. Likewise, in the series mode, the pressure is first released into the capstan followed by the drum motors. The condition will not be satisfied when both the motors of the capstan and drum have same pressure.

Case 4: Troubleshooting is also possible during Pull Out condition. Here, the load cell for tension and proximity sensor for RPM provides the feedback from the system. When the tension is not given during pulling out of the rope and the drum still rotates at a speed, then it is proven that either the check valve or sequence valve is faulty.

Thus, with all these reminders, the winch can remotely troubleshoot and alert the supervisors on site by an email or a short message service (SMS) for immediate technical assistance. Before commencing operation of the cable pulling winch, the operators set definite values of the run-time of the machine. This timing is the service time of the machine. To conduct predictive maintenance, the machine self-evaluates and monitors its internal system. Once the working of the machine exceeds given service time, the staff are alerted to process replacements of hardware and working fluid if necessary. The procedures of M2M connection is required to necessitate the remote control monitoring of the cable pulling winch. Figure 9 shows the necessary elements to conduct this specific task of remote control and monitor.

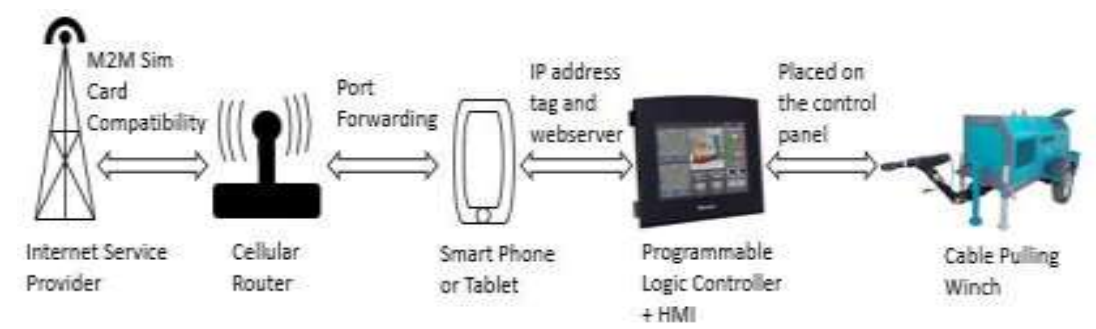

Fig. 9 Block diagram of the steps to understand while implementing M2M connection for a winch

To elucidate further, the first step is to attain an internet service provider (ISP) that can provide the organization with a specific type of connectivity data plan. The carriers will issue an M2M compatible SIM card that has the competence to send data and messages (Emails/SMS) over a network. The Internet is a switching network used in a wide area network (WAN) and is delivered in packets through an Ethernet or local area network (LAN) connection to multiple devices interconnected over the same network. In this case, the internet is delivered via a Sim Card with a 3G/ UTMS (3rd generation, universal telecommunication mobile services) network as it is supported by the cellular router. A secure VPN communication is established between the ISP, Mobile, and Router.

This particular SIM card in Figure 10 is called Machine Identification Module (MIM). This $3 \mathrm{G}$ network provides impressive speed due to code division multiplexing (CDMA) reduces latency and increases the speed of data transfer due to a wider radio spectrum. Once installed in a cellular router, IP addresses are assigned to devices via dynamic host configuration protocol (DHCP) that need to connect over a single network. This address acts as a tag to open a gateway between devices. The process of remapping the destination IP address or redirecting a request from one address and port number to another under a router is called as Port Forwarding. 


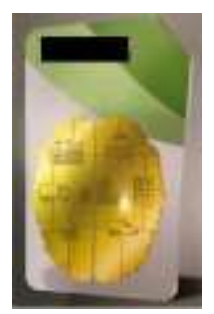

Fig. 10 M2M Sim Card inserted in a cellular router

This cellular router uses network address translation (NAT) thus allowing or translating IP addresses of multiple digital devices to appear on the Internet. As IP addresses mimic web pages, Domain name service (DNS) maps addresses to websites. The cellular router is equipped with various ports. The main ports include Ethernet connection, RS232 serial communication, and USB port. It is powered by a DC supply through an RJ25 (6 port registered jack) cable and connected to the PLC via an RS232 serial communicator cable. RS232 is a short range, point to point communication is between a data terminal equipment (DTE) and a data communication equipment (DCE) [7]. The Sim Card requested must have a static IP address, GPRS username and password and an access point name username and password (APN or wireless access point (WAP)). A static IP is chosen as several machines are manufactured. Every time a device reconnects to a public network, the address resets. Hence, a private or reserved ID is used for each machine to eliminate confusion.

\section{CONCLUSION}

The research paper is categorized into chapters that extrapolate topics of a mode of communication and data transfer. As it is necessary that there must be a continuous flow and feedback of data like texts, emails, remote access and file transfers, TCP/IP is inculcated in this case. Utilizing a PLC with an embedded web server allows the user to store data in a single system rather than a separate expensive cloud base system. This paper also establishes the method of IoT implementation and all the advantages in a hydraulic machine i.e. the cable pulling winch machine. Real-time testing performed with successful results include:
a) Remote control and monitoring
b) Remote troubleshooting
c) Predictive maintenance

With an advanced mode of interoperability discussed in accordance to M2M cellular connectivity and embedded sensors, conducting these experiments proved to be beneficial, a feasible human-technology interaction, secured over a virtual private network and simplified to expose the product to the market.

\section{REFERENCES}

[1] Mehdia. A \& M. Boulmalf. "Smartphone: The Ultimate IoT and IoE Device." Smart phones from an Applied Research Perspective. 2017. pp.137-162.

[2] Julian Marius M, Daniel K \& Kai I.V. "What drives the implementation of Industry 4.0? The Role of Opportunities and Challenges in the Context of Sustainability." Sustainability. 2018. vol.10.

[3] Yoga Liu, Rung-Huei Liang, Ya-Han Lee, Yaliang Chuang \& Lin-Lin Chen. "Designing the Expressiveness of Point Lights for Bridging Human-IoT System Communications." in Proceedings of the Conference of Design and Semantics of Form and Movement. 2017. pp. 165-172.

[4] Robert Nagel \& Stefan Morscher. "Connectivity Prediction in Mobile Vehicular Environments Backed By Digital Maps.” Advanced Trends in Wireless Communications. 2011. pp. 243-267.

[5] Mirjana Simić \& Predrag Pejović. "Positioning in Cellular Networks." Cellular Networks. 2011. pp. 51 - 
78.

[6] Phuoc Nguyen Tran \& Nadia Boukhatem. "IP-based RFID Location System." Radio Frequency Identification Fundamentals and Applications. 2011. pp. 132-147.

[7] Virgilius-Aurelian M. \& Eugen Petac. "A Virtual Routing Solution for IP Networks." Proceedings of the International Conference of Interdisciplinary Studies. 2016. pp. 54-82.

[8] Thienne J., Eleri C., Rodrigo P., E. Zagari \& Tomas B. "Mobility in IP Networks: from Link Layer to Application Layer Protocols and Architectures." Radio Communications. 2010. pp. 573-598.

[9] Meng Z, Wu Z \& John Gray. "A Collaboration-oriented M2M Messaging Mechanism for the Collaborative Automation between Machines in Future Industrial Networks." Smart Industrial Wireless Sensor Networks., 2017. vol. 17. no. 11.

[10] Pereira C. \& Aguiar A. "Towards Efficient Mobile M2M Communications: Survey and Open Challenges" Sensors. 2014. vol. 14. no. 10. pp. 19582-19608.

[11] Kesar M, Samba V, Kinza T, Ram K \& R Udayakumar. “Automation of underground cable laying equipment using PLC and HMI." Proceedings of IOP Conference in Materials, Science and Engineering. 2018. vol. 346. 